\title{
Characterization of the Morphology of Faceted Particles by Transmission Electron Microscopy
}

Shirley Turner, David S. Bright

Chemical Science and Technology Laboratory, National Institute of Standards and Technology, Gaithersburg, MD 20899-8371

\begin{abstract}
Faceting in a polyhedral rutile particle was modeled from transmission electron microscopy images. A double-tilt, rotate transmission electron microscope (TEM) sample holder was used to manipulate the particle. Using this holder, it was possible to align the $\underline{\mathrm{c}}$ axis of the particle along one of the axes of the sample holder. This alignment allowed images to be obtained of the particle in several orientations around its $\underline{\mathrm{c}}$ axis. Comparison of dimensions and angles obtained to those obtained for hypothetical models of the particle gives information about its likely prismatic and pyramidal faceting. This approach to facet modeling is useful for more complete determination of the faceting in individual euhedral particles using transmission electron microscopy.
\end{abstract}

\section{INTRODUCTION}

Identification of the faceting in polyhedral particles is important in many applications. For example, different facets in catalysts have different efficiencies for promoting reactions. In pigment particles, certain morphologies are more desirable for durability of paint.

Characterizing the morphology of particles with transmission electron microscopy is challenging because a TEM image is a two-dimensional (2D) projection of the material. In the biological field, 3D reconstruction techniques have long been used to derive 3D models of cellular and other biological structures from 2D TEM images. Recently, there have been several efforts to apply similar reconstruction techniques in the material science field [1-4]. The techniques have been applied to deriving the distribution of metal particles on silicates [1,2], channels in zeolites [1], and particles in nanocomposites [4].

The 3D reconstruction techniques have not yet been used to derive information about the faceting of particles. At present, such faceting information is typically derived by examining oriented crystallites and measuring angles between linear features in the outline of the 2D projections. If several crystallites are found in different orientations, a 3D model for the typical particle can be derived. Such a 3D model is an average of information from different particles and is not particle specific. In this work, a double-tilt, rotate (2TR) holder is used to orient and tilt a particle to derive information for comparison with morphological models. The 2TR holder has three degrees of freedom theoretically allowing the TEM operator to align a crystallographic direction of interest along one of the tilt axes. This alignment is not possible with conventional double-tilt or rotate-tilt holders unless serendipity leads to a suitably oriented particle. When a crystallographic direction of interest is suitably oriented, tilting of the particle around that direction can allow for measurement of dimensions and comparison to models of crystallite faceting. 


\section{EXPERIMENTAL}

The material examined was rutile $\left(\mathrm{TiO}_{2}\right)$ used in paint pigment. The morphology of rutile particles is affected by $\mathrm{Al}_{2} \mathrm{O}_{3}$ content [5,6]. The material was dry mounted onto a carbon film grid. It was studied in an FEI CM30 TEM operated at $300 \mathrm{kV}^{1}$. A Gatan Model 679 CCD camera was used to record images of the particle. A Gatan Model 925 TEM sample holder was used in the study (note: in this work, the tilt axis along the rod length is referred to as the x axis; the tilt axis perpendicular to the $\mathrm{x}$ axis is referred to as the $\mathrm{y}$ axis). The tilt range around the $\mathrm{x}$ axis is limited by the objective pole piece ( $\pm 45^{\circ}$ in this work) and tilt around the y axis is $\pm 24^{\circ}$. Measurement of particle width and length was done using tools in Lispix, a public domain image analysis program for Windows [7,8]. The particle was highlighted using a threshholding tool and the maximum and minimum diameters determined automatically using a caliper tool. Two sets of measurements were made. Particle modeling was aided by use of Shape software [9].

\section{RESULTS}

For this initial study, a euhedral rutile particle approximately $200 \mathrm{~nm}$ in width and 500 $\mathrm{nm}$ in length was chosen. The particle was oriented down [110] using the double-tilt $\mathrm{x}$ and $\mathrm{y}$ axes of the sample holder. The particle was then rotated around [110] so that the $\underline{\mathrm{c}}$ axis of the particle was aligned along the $\mathrm{x}$ axis of the sample holder (Fig. 1a). The particle was then tilted around its $\underline{\mathrm{c}}$ axis (Figs. 1a-f). The alignment of the $\underline{\mathrm{c}}$ axis of the particle and the $\mathrm{x}$ axis of the sample holder is confirmed by the acquisition of several diffraction patterns containing [001]* including those in Figs. 1b, d, and f.

\section{Prismatic faceting}

For the 2D projection given in Figure 1a, there are several possible prismatic facets including those commonly found for rutile $-\{110\},\{230\},\{120\},\{130\},\{140\},\{170\}$ and $\{100\}[10]$. It is not possible to distinguish between these forms using the single image of Figure 1a. However, the good alignment of the $\underline{\mathrm{c}}$ axis of the particle to the $\mathrm{x}$ axis of the sample holder allows for deductions to be made about the prismatic faceting based on measurement of the particle width and its variation with tilt angle. Images were collected in intervals of $3^{\circ}$ tilt around the particle $\underline{\mathrm{c}}$ axis with the [110] orientation at $0^{\circ}$ tilt. The width of the particle at each tilt was measured. Each width was then divided by the width of the particle in the [110] orientation.

Subsequently, the projected widths for hypothetical particles with the common rutile forms were calculated at $3^{\circ}$ intervals from the [110] orientation. As done for the rutile particle, each width was then divided that of the particle in the [110] orientation. Plots of the width ratios vs. tilt angle for the actual particle and the models are given in Figure 2. The width ratios for the particle do not exactly match any of those for the common single forms. It is therefore postulated that the prismatic facets consist of a combination of forms. We note from

\footnotetext{
${ }^{1}$ Certain commercial equipment, instruments, or materials are identified in this paper to specify adequately the experimental procedure. Such identification does not imply recommendation or endorsement by the National Institute of Standards and Technology, nor does it imply that the materials or equipment are necessarily the best available for the purpose.
} 


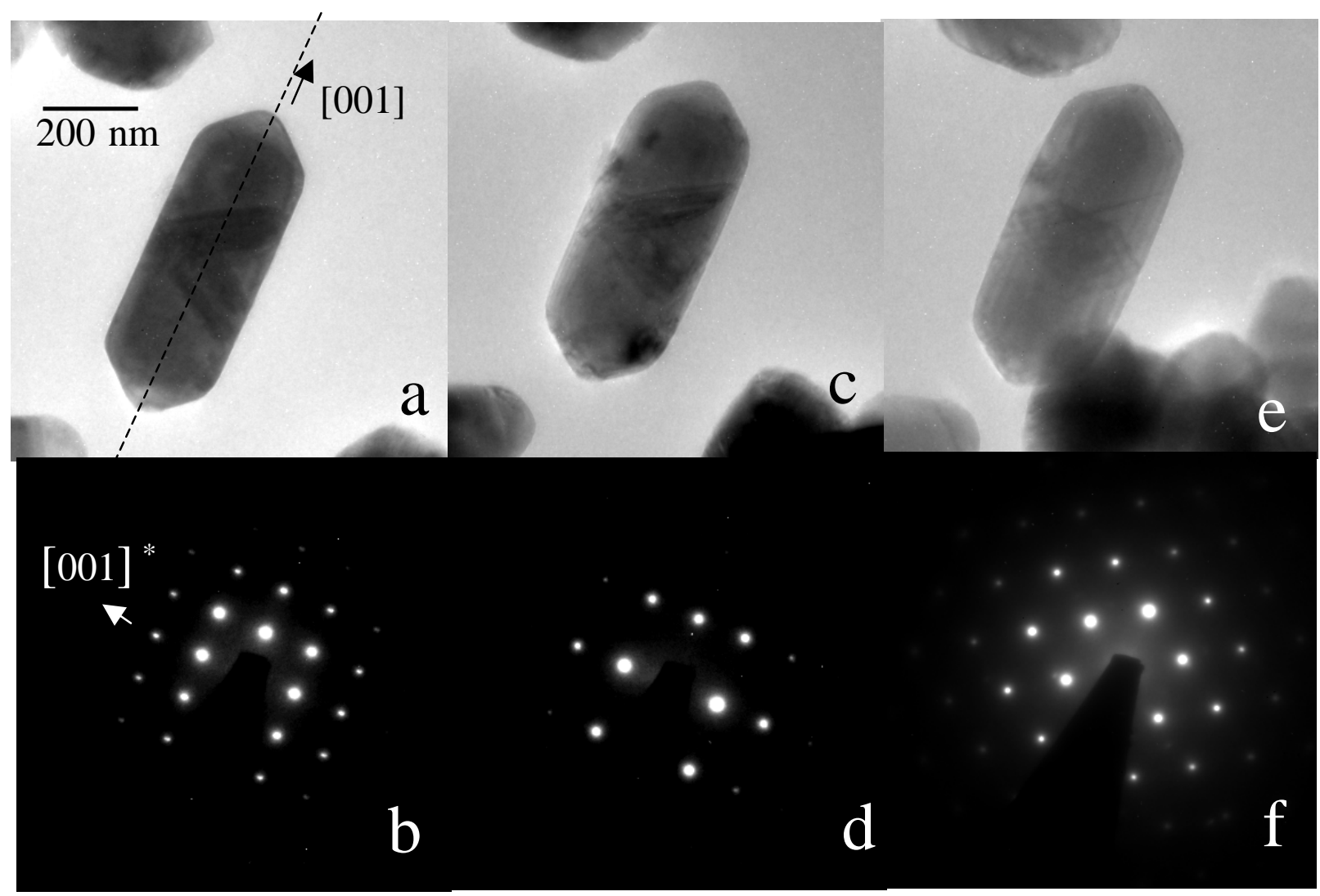

Figure 1. Images and diffraction patterns of a rutile particle. The particle is oriented down [110] so that the $\underline{\mathrm{c}}$ axis of the particle is aligned along the $\mathrm{x}$ axis of the sample holder (projection of $\mathrm{X}$ axis indicated by dashed line in Fig 1a). The particle is rotated around the holder $x$ axis. Diffraction patterns containing [001]* are obtained (b) [110], (d) [210], and (f) [100].

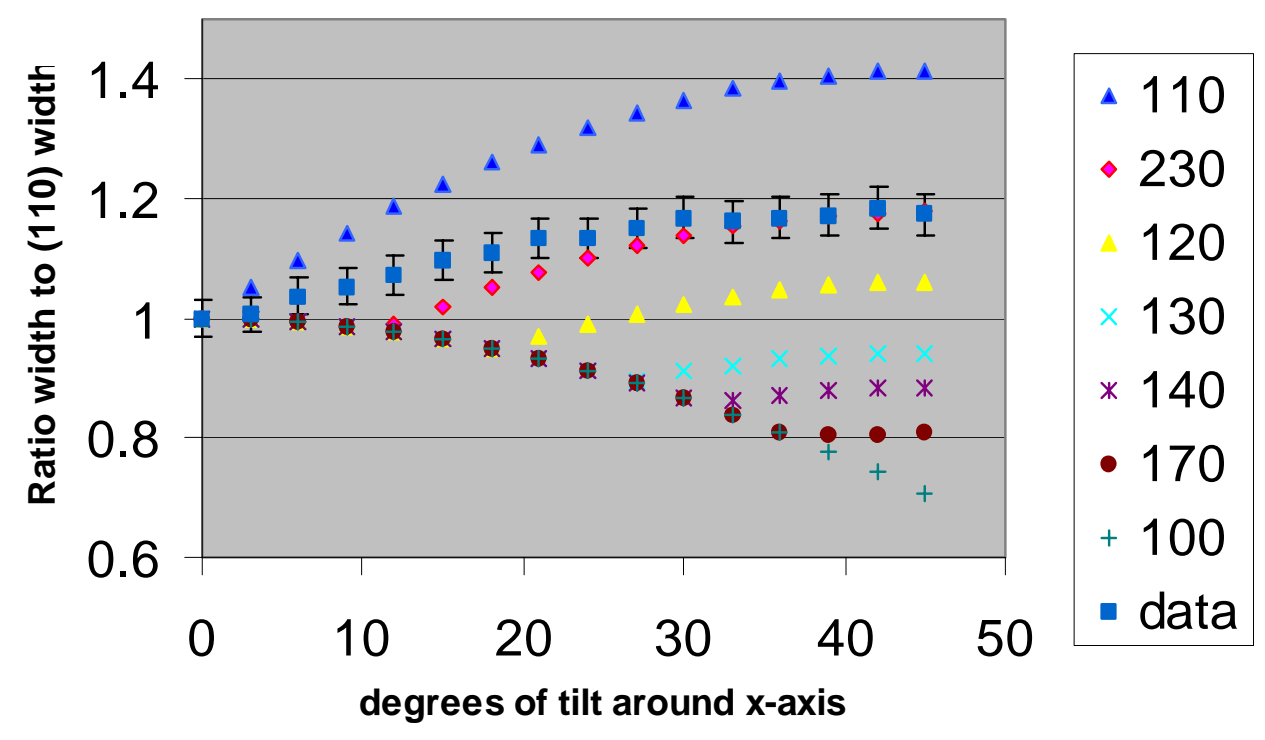

Figure 2. Plots of the ratio of particle width to particle width in [110] orientation vs. degrees tilt for various prismatic forms of rutile and for particle data. 
Figure 2 that the width ratios for the particle increase for increasing tilt angle whereas there is an initial decrease in width ratios for $\{230\},\{120\},\{130\},\{140\},\{170\}$ and $\{100\}$. It is therefore postulated that $\{110\}$ is a major facet since it is the only form for which the width ratio consistently increases with tilt angle.

Possible combinations of common forms include $\{110\}$ with $\{120\},\{130\},\{140\},\{170\}$ or $\{100\}$. Models of three of these combinations are shown in Figure 3 a-c. Width ratios for the five possible combinations were determined for different orientations for the models rotated around the $\underline{\mathrm{c}}$ axis at $3^{\circ}$ intervals (plots for three of the models are given in Figs. $3 \mathrm{~d}-\mathrm{f}$ ). A comparison of the data and plots for the five cases shows that the $\{120\}$ modification is not a good match to the data; the other models show a fairly good match. Given the uncertainties in measurements in the present work, it is not possible to distinguish between the $\{110\}$ form modified by $\{130\},\{140\},\{170\}$ or $\{100\}$.

Pyramidal faceting

Pyramidal facets can be modeled directly from the projected angles (Figs. 4a, b). The average measured pyramidal angles in the [110] orientation is $\sim 95^{\circ} \pm 2^{\circ}$ and in the [100] orientation is $\sim 114^{\circ} \pm 2^{\circ}$. These values can be compared to those expected from pyramidal facets commonly found in rutile (Table 1) [10]. Projected angles were determined from Shape software.

Table 1. Projected angles for common pyramidal facets of rutile

\begin{tabular}{ccc}
\hline Pyramidal form & Projected angle [110] & Projected angle [100] \\
\hline$\{101\}$ & $131.02^{\circ}$ & $114.42^{\circ}$ \\
$\{111\}$ & 95.38 & 114.42 \\
$\{301\}$ & 72.39 & 54.72 \\
$\{501\}$ & 47.41 & 34.5 \\
$\{221\}$ & 57.52 & 75.63 \\
$\{133\}$ & 117.46 & 114.42 \\
$\{131\}$ & 57.52 & 54.72 \\
$\{233\}$ & 105.59 & 114.42 \\
$\{231\}$ & 47.13 & 54.72 \\
\hline
\end{tabular}

Projected angles for $\{111\}$ most closely match the angles in experimental images in both [110] and [100] orientations. Note that for both the [110] and [100] orientation there are forms with the same projected angles. Acquisition of angles in two orientations is helpful in making an unambiguous identification.

Model for faceting of the particle

A basic model for faceting of the particle consists of $\{110\}$ prismatic facets with $\{111\}$ pyramidal facets. The corners of the $\{110\}$ facets are truncated. A working model of the faceting is shown in Figure 5 in the [110] and [100] orientation. The modification of $\{110\}$ is represented as $\{\mathrm{hk} 0\}$. The model is an approximation to the particle shape as there is slight curving of the crystallite edges and rounding of facet corners in some projections. 


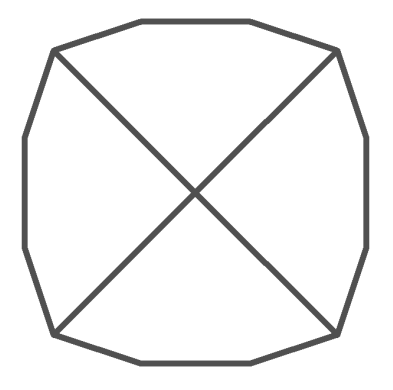

$$
\{110\}+\{120\}
$$

a

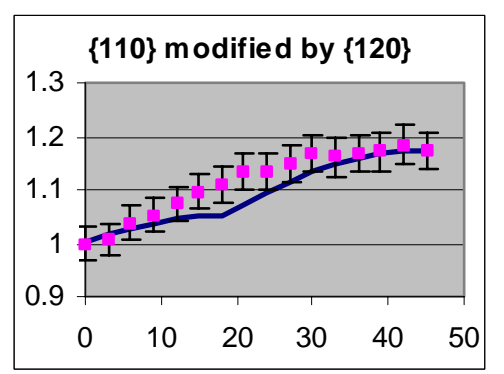

d

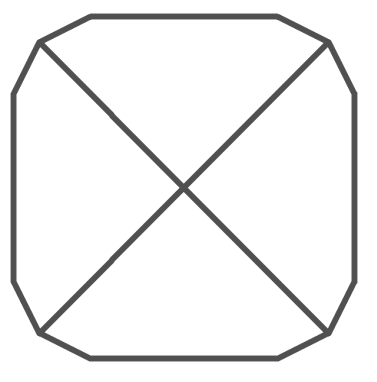

$\{110\}+\{140\}$

b

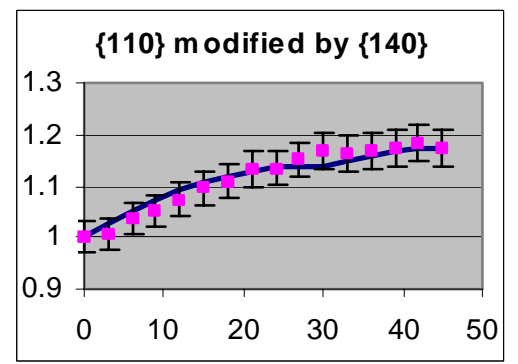

$\mathrm{e}$

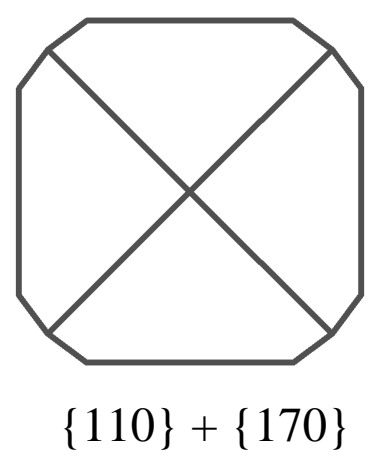

C

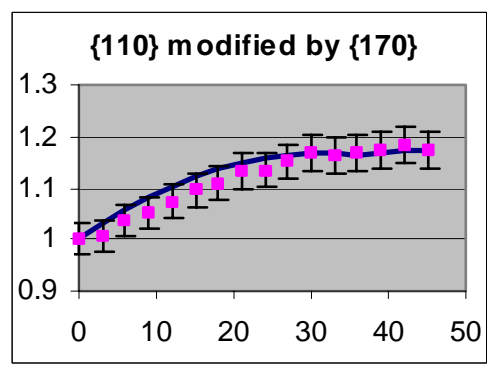

$\mathrm{f}$

Figure 3. (a-c) Examples of three possible combinations of common prismatic rutile forms. The models are viewed down [001]. The dimensions of the facets fit the width ratios for data collected from the particle in the [110] and [100] orientations. (d-f) Plots of projected width ratio (y axis of plot) vs. tilt angle ( $\mathrm{x}$ axis) for tilting of each model above. The lines on the plots correspond to the width ratio for the model; the markers correspond to data from the particle.
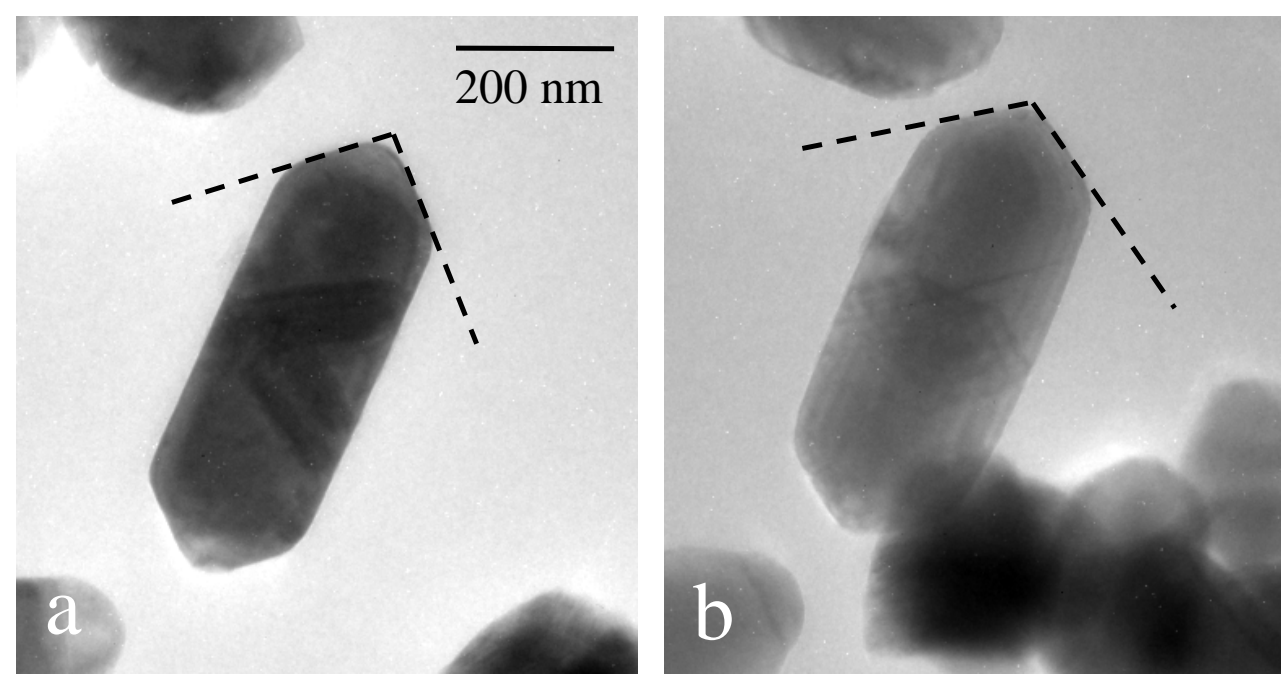

Figure 4. Projected pyramidal angles (dashed lines) of rutile particle in (a) [110] orientation and (b) [100] orientation. 

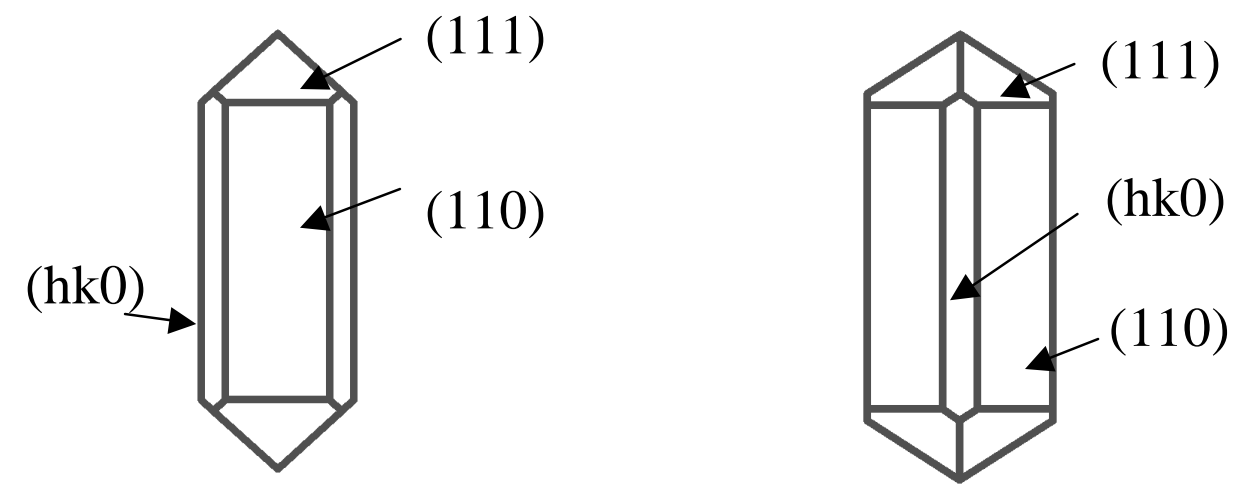

a

\section{b}

Figure 5. Model for faceting of rutile particle shown in (a) [110] and (b) [100] orientations.

\section{SUMMARY}

This work has shown that the 2TR holder allows for alignment of crystallographic directions of interest along holder axes. Measurement of changes in crystal width and angles in a range of known orientations can therefore be compared to changes in widths and angles expected from possible models of crystal faceting in the same orientations. In this initial study of a rutile particle, measurement of width ratios indicates that $\{110\}$ is the likely predominant prismatic facet. The width ratio data also indicates that the $\{110\}$ faceting is further modified although the uncertainty in the measurement does not allow distinction between several facet models. Measurement of projected angles of the particle in two orientations indicates that $\{111\}$ is the predominant pyramidal facet. Basic morphological information has been derived about the particle in the study, however, the faceting model is an approximation to the actual shape. It is expected that this approach to morphology determination in combination with the 3D reconstruction techniques recently applied in the material sciences will be a useful approach to characterizing faceted, euhedral particles.

\section{REFERENCES}

1. A.J. Koster, U. Ziese, A.J. Verkleij, A.H. Janssen, and K.P. De Jong, J. Phys. Chem. B, 104 (40), 9368 (2000).

2. M. Weyland, P.A. Midgley, and J.M. Thomas, J. Phys. Chem. B. 105 (33), 7882 (2001).

3. P.A. Midgley, M. Weyland, J.M. Thomas and B.F.G. Johnson, Chem. Commun, 2001, 907 (2001).

4. G. Mobus and B.J. Inkson, Appl. Phys. Let. 79 (9), 1369 (2001).

5. U. Gensenhues, Solid State Ionics 101-103, 1171 (1997).

6. U. Gensenhues and T. Rentschler, J. Sol. State Chem. 143, 210 (1999).

7. D.S. Bright, Microbeam Analysis 4, 151 (1995).

8. www.nist.gov/lispix/2

9. www.shapesoftware.com

10. C. Palache, H. Berman and C. Frondel, Dana's System of Mineralogy, Vol. 1, (John Wiley and Sons, New York, 1944).

\footnotetext{
${ }^{2}$ At the time this paper was written, URLs referenced herein were deemed to be useful supplementary material for this paper. Neither the author nor the Materials Research Society warrants or assumes responsibility for the content or availability of URLs referenced in this paper.
} 